\title{
A new grounding model for complex soil environments
}

\author{
Chong Pan ${ }^{1}$, Dong Zhao ${ }^{1}$, Xuesong Tu${ }^{1}$, Shuqin Wang ${ }^{1}$, Tie Liu ${ }^{1}$ and Yiqiao $\mathrm{Li}^{2}$ \\ ${ }^{1}$ Maanshan Power Supply Company, State Grid Anhui Electric Power Co.,Ltd, Maanshan Anhui, 243000, China \\ ${ }^{2}$ State Key Laboratory of Power Transmission Equipment \& System Security and New Technology, Chongqing University, Shapingba, \\ Chongqing, 400030, China
}

\begin{abstract}
The purpose of arranging the grounding electrode is to discharge large currents, such as fault current and ensure the transmission line operate safely. In the grounding system of Chinese transmission network, the common grounding electrodes are horizontal installation and vertical installation. But two installation methods usually have the disadvantages, which are long distance installation and large depth burying, and they are restricted susceptible to uncertain factors such as pipelines, roads and geology, which leads the grounding resistance increasing and affect the normal operation of transmission lines. In order to solve the problem, this paper presents a new grounding model for complex soil environments and analysis from grounding resistance, surface potential and current distribution. The results show that the helix grounding electrode has good resistance reduction performance in complex soil environment, which effectively improves the surface potential distribution and the distribution of the spill current.
\end{abstract}

\section{Introduction}

The grounding system is an indispensable component of the power system. Some parts of the electrical equipment and a certain point of the power system are connected to the earth through the electrical connection terminal. It provides a discharging channel for the fault current to ensure the potential unable to reach the upper limit which poses a danger to the human body, the personal safety of the power system operators and the safe operation of electrical equipment[1]. The grounding electrode is an important part of the grounding system. At present, the conventional grounding electrodes are as follows: natural grounding electrode, closed loop grounding electrode, horizontal grounding electrode and vertical grounding electrode. For example, the basic steel mesh frame can be fully utilized as a natural grounding electrode in wet areas with soil resistivity $\rho \leq 100$ and areas with operational experience; the grounding device should surround the tower foundation laid as a closed loop in residential areas, paddy fields and other horizontal grounding electrode where restrictions are imposed; the grounding device uses a radial grounding electrode or a continuously elongated grounding electrode in a wider area; Several copper rods, steel rods and angle steel are penetrate into the ground vertically as the grounding electrode in the steeper western China. The above conventional installation methods have disadvantages[2-3]. For example, the natural grounding electrode has the problem that the current discharge is difficult to meet the expected requirements; the closed loop grounding device has a deep buried depth and the applicable range is limited; the long length of horizontal grounding electrode increases the construction difficulty and cost. The vertical grounding electrode has the problem of uneven current discharge and high soil temperature rise.

The grounding performance of conventional grounding electrodes in complex soils can be improved by changing soils locally, resistance reducing agent, and increasing the contact area between the grounding electrode and the soil. It is theoretically feasible to reduce the soil resistivity by local soil replacement, and it is possible to replace the soil with high soil resistivity. However, in the actual construction process, the soil type in the mountainous area is relatively complicated, and the partial soil replacement can only replace the surface soil. For the lithosphere and the gravel bed, the engineering amount of replacing the soil is large, and the implementation cost is high. The addition of a resistance reducing agent can reduce the soil resistivity around the grounding electrode through its diffusion and osmosis. However, the long-term use of resistance reducing agent in practical engineering applications can lead to a series of problems, such as corrosive problems, stability problems and pollution of groundwater resources. Comprehensive economics and practicability, among the many means to improve the grounding performance, the most direct and effective way is to increase the contact with the soil, usually by increasing the horizontal grounding electrode and vertical grounding electrode length.

All of the above studies have focused on improving the grounding electrode property by reducing the soil resistivity. Few scholars have proposed a new grounding electrode structure when the grounding performance is not satisfied. At present, the commonly used horizontal and vertical grounding electrodes are very vulnerable to

\footnotetext{
*Corresponding author: 641139455@qq.com
} 
terrain, and the grounding performance is seriously degraded in areas where the long distance is not possible. In order to improve the grounding performance of the grounding electrode in complex soil environment, based on the idea of expanding the contact area between the grounding electrode and the soil in the unit axial space, this paper proposes a grounding electrode model of the helix structure and analyzes its grounding performance.

\section{Finite element basic theory}

The finite element method is the most widely used in the numerical calculation of electromagnetic fields. The traditional finite element method is based on the variation principle. The boundary value problem of the required solution is transformed into the corresponding variation problem, and then variation problems are discretized into extremum problems of ordinary multivariate functions by using the interpolation. The function of the grounding electrode is to discharge the fault current, and the current discharges into the soil through the grounding electrode. The space potential equation of the grounding electrode is usually used in engineering to analyze the grounding system's current discharge process. When the current is discharged through the grounding electrode, the grounding electrode can be approximated as an equipotential body. In order to solve the potential of any point in the space in infinite earth, the grounding electrode is usually regarded as a set of point current sources, so the potential function $\varphi(x, y)$ satisfies the differential equation[4-5].

$$
\nabla^{2} \varphi+\rho_{s} I \delta\left(\vec{r}-\overrightarrow{r^{\prime}}\right)=0
$$

Where, $I$ is the size of the point current source in the field, $\vec{r}$ represents the spatial position of the field point's vector, and $\vec{r}$ represents the spatial position's vector of the source point. $\delta\left(\vec{r}-\overrightarrow{r^{\prime}}\right)$ is the Dikelac function, $\rho_{s}$ is the soil resistivity.

The current discharges through the grounding electrode within a certain range of the soil. If the infinity is used as the zero potential, the computer's computational efficiency are greatly reduced and the memory requirement is increased. In order to overcome the above shortcomings, this paper uses finite element analysis to coordinate the open space outside the selected area. In order to simulate the zero potential point at infinity, the radius of the hemispherical field of the simulated soil should be at least 5 times the axial length of the grounding electrode. The equivalent method effectively avoids the inconvenience caused by directly solving the open space, and greatly improves the efficiency of the solution.

If $\Omega$ is set as the field to be obtained, $\Gamma_{1}$ is the interface between the soil medium and the air, $\Gamma_{2}$ is simulate the equivalent boundary at infinity zero potential, and the boundary is $\Gamma=\Gamma_{1}+\Gamma_{2}, \Gamma_{3}$ is the interface between the surface of the grounding electrode and the soil medium. When the voltage drop of the grounding electrode surface is ignored, the approximate helix ground potential surface potential is constant $\varphi_{0}$. The boundary value problem can be listed:

$$
\left\{\begin{array}{l}
\nabla^{2} \varphi=-\rho_{s} I \delta\left(\vec{r}-\overrightarrow{r^{\prime}}\right) \in \Omega \\
\left.\varphi\right|_{\Gamma_{2}}=0 \\
\left.\varphi\right|_{\Gamma_{3}}=\varphi_{0} \\
\left.\frac{\partial \varphi}{\partial n}\right|_{\Gamma_{1}}=0
\end{array}\right.
$$

According to the above formula, the grounding electrode current discharging analysis process is a Poisson boundary value problem, which can convert the typical boundary value problem into an equivalent variation problem.

$$
\left\{\begin{array}{l}
J\{\varphi\}=\frac{1}{2} \int_{\Omega} \frac{1}{\rho_{s}}(\nabla \varphi)^{2} d \Omega-\int_{\Omega} I \delta\left(\vec{r}-\overrightarrow{r^{\prime}}\right) \varphi d \Omega=\min \\
\left.\varphi\right|_{\Gamma_{2}}=0 \\
\left.\varphi\right|_{\Gamma_{3}}=\varphi_{0}
\end{array}\right.
$$

It can be seen from the above equation that there is no third term in the variation equation, and the variation problem of the current dispersion of the grounding electrode in the soil satisfies the second homogeneous boundary condition. The processing of variation equations satisfying the second kind of homogeneous boundary conditions is often to transform them into a rectangular coordinate system to write their functional expressions, then mesh and interpolate the threedimensional field, and finally sort out finite element equation.

In rectangular coordinates, the functional is:

$$
F(\varphi)=\frac{1}{2} \int_{\Omega} \frac{1}{\rho_{s}}\left[\left(\frac{\partial \varphi}{\partial x}\right)^{2}+\left(\frac{\partial \varphi}{\partial y}\right)^{2}\right] d x d y-\int_{\Omega} I \delta\left(\vec{r}-\overrightarrow{r^{\prime}}\right) \varphi d x d y
$$

For the meshing and interpolation of the threedimensional field, the first-order tetrahedral element is generally selected as the basic unit of the threedimensional field. If it is assumed that the solved field is divided into Z0 units by the tetrahedron, N0 discrete nodes are obtained. Each node corresponds to a unique space potential equation, taking the four vertices of the first-order tetrahedral element as nodes, which correspond to the actual number in the whole domain is $i, j, l, m$.

According to the linear interpolation is $\tilde{\varphi}(x, y, z)=\alpha_{1}+\alpha_{2} x+\alpha_{3} y+\alpha_{4} z$, the interpolation function is continuous in the unit tetrahedron $e$, and respectively brings in the coordinates of the four vertices 
and the corresponding bit values, and obtains: $\alpha_{1}+\alpha_{2} x_{i}+\alpha_{3} y_{i}+\alpha_{4} z_{i} \quad i=(1,2,3,4)$.

The simultaneous equations are solved for each undetermined coefficient $\alpha_{1}, \alpha_{2}, \alpha_{3}, \alpha_{4}$ and brought them into the interpolation function.

$$
\tilde{\varphi}(x, y, \mathrm{z})=N_{1} \varphi_{1}+N_{2} \varphi_{2}+N_{3} \varphi_{3}+N_{4} \varphi_{4}=\sum N_{i} \varphi_{i}=[N]_{e}^{T}[\varphi]_{e}
$$

To form a finite element equation, the elements of the coefficient matrix in the rectangular coordinate system are required:

$k_{k p}^{e}=\int_{V_{e}} \frac{1}{\rho_{s}} \nabla N_{k} \cdot \nabla N_{p} d v=\int_{V_{e}} \frac{1}{\rho_{s}}\left[\frac{\partial N_{k}}{\partial x} \frac{\partial N_{p}}{\partial x}+\frac{\partial N_{k}}{\partial y} \frac{\partial N_{p}}{\partial y}+\frac{\partial N_{k}}{\partial z} \frac{\partial N_{p}}{\partial z}\right] d x d y d z$

$$
\begin{aligned}
& \text { Because } \frac{\partial N_{k}}{\partial x}=\frac{b_{k}}{6 V_{e}}, \frac{\partial N_{k}}{\partial y}=\frac{c_{k}}{6 V_{e}}, \frac{\partial N_{k}}{\partial z}=\frac{d_{k}}{6 V_{e}}, \text { so : } \\
& k_{k p}^{e}=\frac{1}{36 \rho_{s} V_{e}}\left(b_{k} b_{p}+c_{k} c_{p}+d_{k} d_{p}\right)(k, p=i, j, l, m)
\end{aligned}
$$

After the splitting and interpolation, the unit analysis of the unit tetrahedron is also required. The matrix elements of the unit analysis $[P]_{e}$ :

$$
p_{k}^{e}=\int_{\Omega_{e}} I \delta\left(\vec{r}-\overrightarrow{r^{\prime}}\right) N_{k} d x d y d z \quad(l=i, \mathrm{j}, \cdots, m)
$$

Substituting the coordinates of the center of the tetrahedron's gravity $\left(x_{c}, y_{c}, z_{c}\right)$ into the above formula, and setting the endogenous density of the element to be approximately unchanged, it is obtained:

$$
N_{k}\left(x_{c}, y_{c}, z_{c}\right)=\frac{1}{6 V_{e}}\left(a_{k}+b_{k} x_{c}+c_{k} y_{c}+d_{k} z_{c}\right)=\frac{1}{4}
$$

Integrate the parameters in the rectangular coordinate system to obtain relevant parameters in the global scope:

$$
[k]=\sum[k]^{e},[p]=\sum[p]^{e}
$$

Therefore, the finite element equation of the Poisson field satisfying the second kind of homogeneous boundary condition is

$$
\begin{aligned}
& {[k][\varphi]-[p]=0} \\
& \Rightarrow[k][\varphi]=[p]
\end{aligned}
$$

\section{Grounding performance analysis}

\subsection{Simulation model construction}

In the actual power transmission and transformation process, the ground current is mainly composed of threephase unbalanced current, and the frequency of the steady-state ground current is $50 \mathrm{~Hz}$. When analyzing the electric field distribution around the grounding electrode, it can be approximately equivalent to a constant electric field. In order to simplify the calculation and ignore the inductance effect at low frequency, the excitation of the grounding electrode selects 1A DC as the grounding current.The hemispherical model as shown in Fig. 1,2,3, which is set up to simulate infinitely uniform soil, and the spherical potential of the hemisphere was set to be zero to simulate the zero potential at infinity. To ensure the calculation accuracy, the radius of the hemisphere should be at least 5 times longer than the axial pole length along the $\mathrm{x}$ direction of the grounding electrode[2].

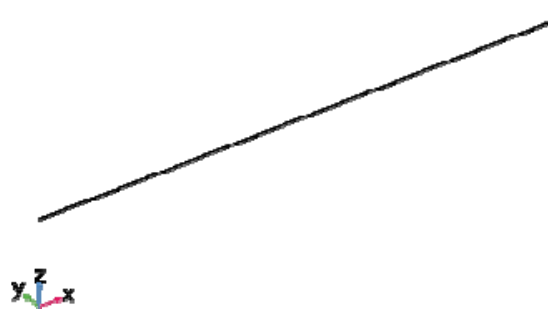

Fig. 1. Horizontal grounding electrode model.

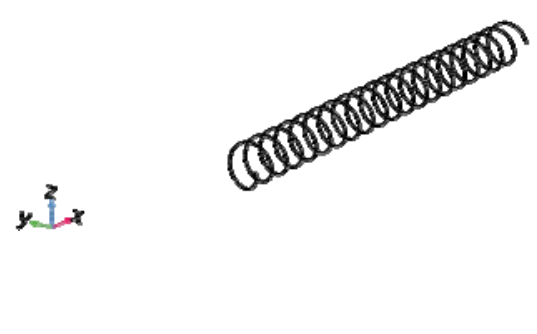

Fig. 2. Helix grounding electrode model.

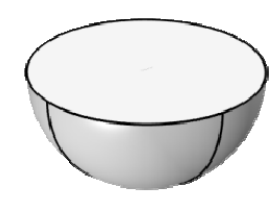

$x^{2}-x$

Fig. 3. Infinite soil model.

\subsection{Grounding resistance}

The grounding resistance of the grounding electrode reflects the current discharge property of the grounding electrode and is one of the important parameters for measuring the grounding performance of the grounding electrode. The horizontal grounding electrode is laid at a 
length of about $10 \mathrm{~m}$ to $20 \mathrm{~m}$. Therefore, a $15 \mathrm{~m}$ horizontal round steel grounding electrode, a $15 \mathrm{~m}$ helix grounding electrode and a $94.2 \mathrm{~m}$ horizontal grounding electrode with the same soil contact area are divided into three groups for comparison. The buried directions are all along the $\mathrm{x}$-axis, and the buried depth is $0.8 \mathrm{~m}$. The volume parameters are presented in Table 1.

Table 1. Grounding electrode structure parameters (unit: $\mathrm{m}$ )

\begin{tabular}{|c|c|c|c|c|c|c|}
\hline & $\begin{array}{l}\text { Axial } \\
\text { length }\end{array}$ & $\begin{array}{l}\text { Sectional } \\
\text { diameter }\end{array}$ & $\begin{array}{l}\text { Helix } \\
\text { coil } \\
\text { radius } \\
\end{array}$ & Turn & Pitch \\
\hline 1 & $\begin{array}{l}\text { Horizontal } \\
\text { round steel } \\
\text { grounding } \\
\text { electrode }\end{array}$ & 15 & 0.012 & & & \\
\hline 2 & $\begin{array}{l}\text { Horizontal } \\
\text { round steel } \\
\text { grounding } \\
\text { electrode }\end{array}$ & 94.2 & 0.012 & & & \\
\hline 3 & $\begin{array}{l}\text { Helix } \\
\text { ground } \\
\text { electrode }\end{array}$ & 15 & 0.012 & 0.5 & 30 & 0.5 \\
\hline
\end{tabular}

By simulating related parameters, the grounding resistance of the helix grounding electrode and the horizontal grounding electrode at the same laying length can be obtained; comparing the Group 2 and the Group 3, the grounding resistor of the helix grounding electrode and the horizontal grounding electrode under the same soil contact area can be obtained.

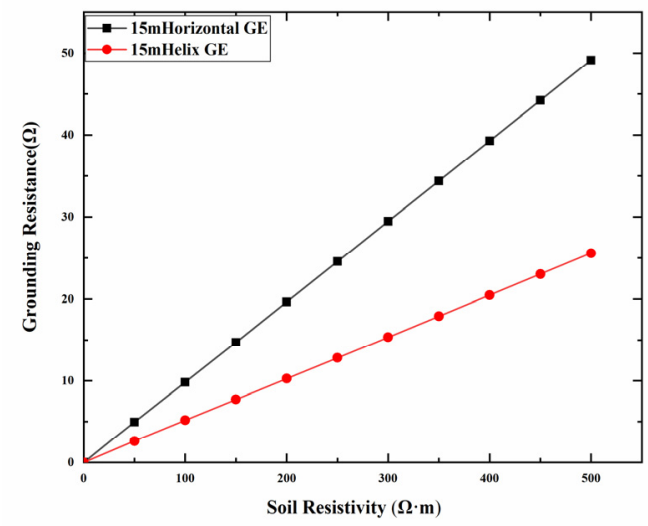

Fig. 4. 15m horizontal grounding electrode and $15 \mathrm{~m}$ helix grounding electrode.

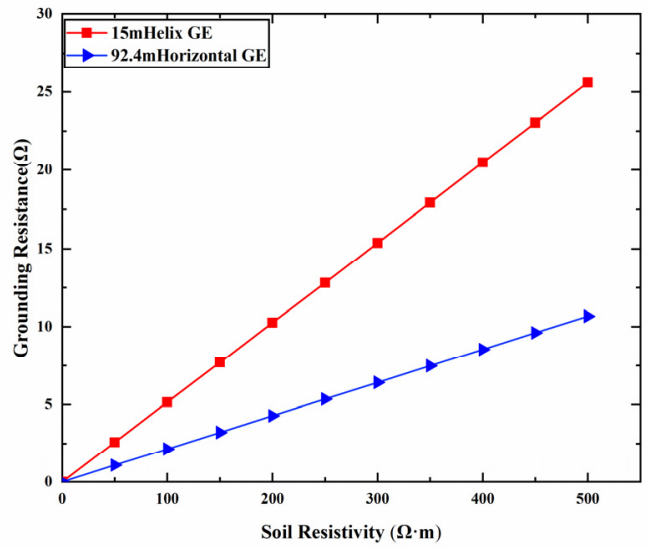

Fig. 5. $15 \mathrm{~m}$ helix grounding electrode and $92.4 \mathrm{~m}$ horizontal grounding electrode.

According to the Fig.4 and Fig.5, the grounding resistance of the three sets of grounding poles increases with the soil resistivity increasing. It can be seen from Fig. 4, that the change rate of the grounding resistance of the $15 \mathrm{~m}$ horizontal grounding electrode is higher than the resistance of the grounding resistance of the helix grounding electrode under the same installation length, indicating that the $15 \mathrm{~m}$ horizontal grounding electrode is extremely susceptible to the soil environment. The grounding resistance of the soil environment of 500 $\Omega \cdot m$ has reached $49.1 \Omega$, which is more than twice the grounding resistance of the helix grounding electrode. In the soil with higher resistivity, the corresponding rent reduction measures must be taken. It can be seen from Fig.5, that the grounding resistance of the $94.2 \mathrm{~m}$ horizontal grounding electrode in the soil with different resistivity is smaller than the helix grounding electrode, but the grounding resistance of the two is not much different. From the perspective of construction cost and operation and maintenance, the $94.2 \mathrm{~m}$ horizontal grounding electrode is much higher than the helix grounding electrode in material cost. The excessively long grounding electrode also brings great difficulties for later maintenance. Long horizontal grounding electrodes will not be applied in actual engineering.

In the complex soil environment, the helix grounding electrode has the advantages of small axial space and good grounding performance, which greatly improves the grounding performance of the grounding electrode in the complex soil environment in the same cloth space. According to the widely used horizontal grounding resistance calculation formula $R=\frac{\rho}{2 \pi l}\left(\ln \frac{l^{2}}{h d}+A\right)$, the horizontal grounding length required for the same value of the grounding resistance of the helix grounding electrode in Table 1 under different soil resistivity is calculated. 


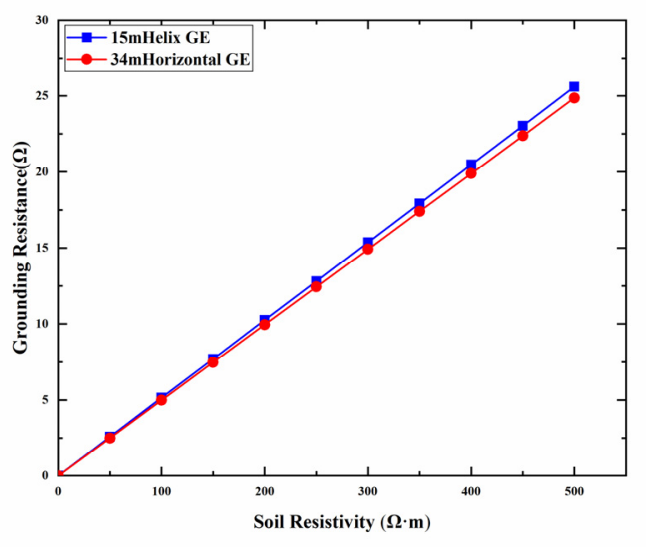

Fig. 6. Horizontal grounding electrode with the same grounding resistance

Fig. 6 shows that the grounding resistance of the helix grounding electrode with a length of $15 \mathrm{~m}$ and the horizontal grounding electrode of the $34 \mathrm{~m}$ is almost the same. Therefore, in a complex soil environment, the helix grounding electrode has better grounding performance and can be better adapted to complex soil environments.

\subsection{The surface potential}

The surface potential directly affects the step voltage and the contact voltage. Both of them are the reasons of personal safety accidents. In addition, the distribution of surface potential will directly affect the corrosion of metal pipes, DC bias of transformers and radio interference, so the surface potential is an important indicator to measure the grounding performance. The ground potential of group 1 and group 3 of grounding electrodes is calculated. The results are presented in the figures below.

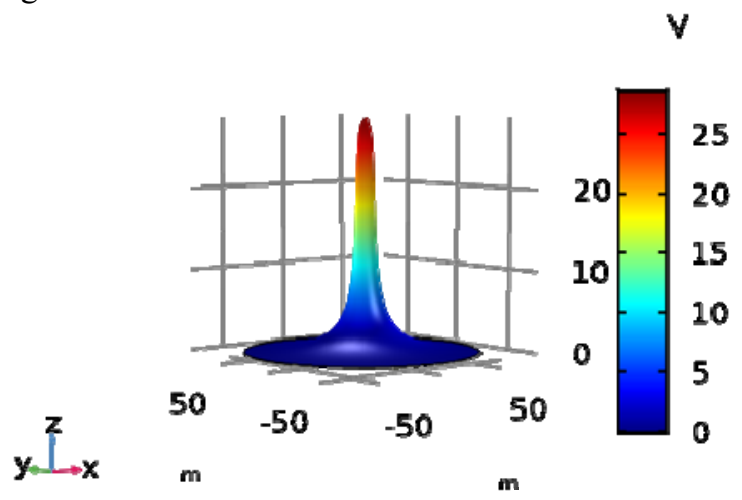

Fig. 7. The Surface potential of $10 \mathrm{~m}$ horizontal grounding electrode.

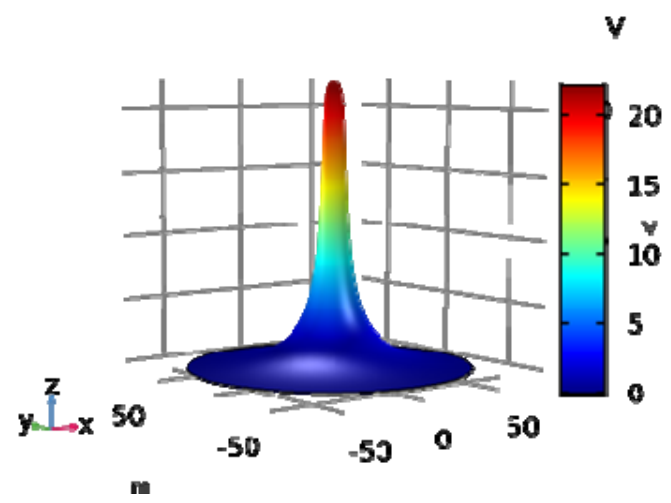

Fig. 8. The surface potential of helix grounding electrode.

According to Fig.7 and Fig.8, the surface potential rises of the two sets of grounding electrodes are concentrated directly above the grounding electrode, and the surface potential drops rapidly near the grounding electrode. The downward trend gradually slows down with the increase of distance, and the ground potential distribution at a distance from the grounding electrode is almost unaffected by the grounding electrode. The maximum ground potential of the helix grounding pole rises to $22.3 \mathrm{~V}$ and the horizontal grounding of $15 \mathrm{~m}$ is $28.8 \mathrm{~V}$, which is about $22.6 \%$ lower than the horizontal grounding pole.

Under the same installation length, the ground potential of the helix grounding electrode is lower, which has higher safety and reliability. Compared with the horizontal grounding electrode, the helix grounding electrode has more advantages in the complex soil environment.

\subsection{Discharging current distribution}

The main function of the grounding electrode is discharging current, which ensures the stable operation of the transmission system and power equipment. Only the grounding electrode with good dispersion ability can discharge large fault currents in the soil in time to ensure reliable operation of electrical equipment. When the ground current is $1 \mathrm{~A}$, the ground current surface discharging current distribution in the first group and the third group is calculated, and the result is as shown in the following figure.

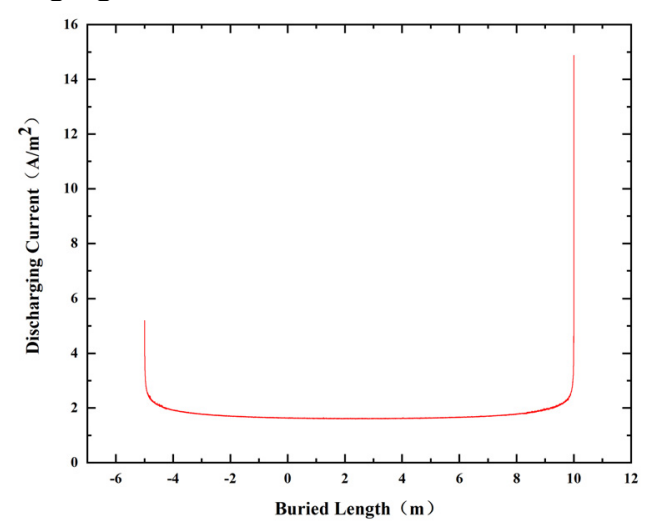

Fig. 9. $15 \mathrm{~m}$ horizontal grounding electrode discharging current distribution 
It can be seen from Fig. 9 that the discharging current is abrupt at the horizontal grounding electrode $x=-5 m$, that is, the grounding electrode is close to the downconducting line, and suddenly drops from $5.07 \mathrm{~V}$ to $1.65 \mathrm{~V}$ and remains substantially unchanged on the grounding electrode surface. That is the end effect of the horizontal grounding electrode. As a whole, the horizontal grounding electrode does not have much or no discharging current at the lower line. In the complex soil environment, the horizontal grounding electrode does not have excellent current discharge property.

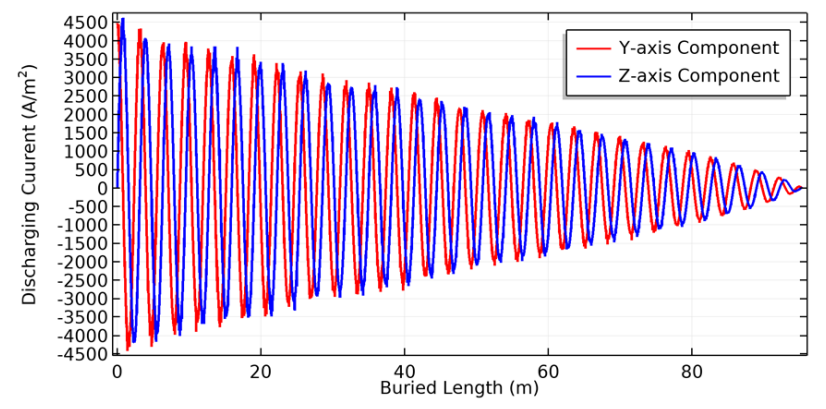

Fig.10 Y-axis Z-axis current discharge distribution of $15 \mathrm{~m}$ helix grounding electrode

It can be seen from Fig.10 that the discharging current of the helix grounding electrode has a tendency to attenuate on the surface, which is the largest at the lead wire and the smallest at the end of the grounding electrode. On the whole, the helix grounding electrode's discharging current is much larger than the horizontal grounding electrode, and the distribution is relatively uniform. The use of a helix grounding electrode in a complex soil environment can effectively increase the efficiency of the discharging current per unit length and provide superior current discharge.

\section{Conclusion}

In the complex soil environment, the existing grounding electrode grounding performance is seriously degraded and cannot meet the engineering requirements. Adopting various measures to reduce the resistance has greatly increased the input cost in the early stage and the operation and maintenance cost in the later stage. In order to solve the above problems, this paper proposes a new grounding model suitable for complex soil environment, namely helix grounding electrode. Based on the finite element method, the helix grounding electrode can be analyzed in detail from three aspects: grounding resistance, surface potential and current distribution. The results are as follows:

(1)In soil media with different resistivity, the grounding resistance of the helix grounding electrode is significantly smaller than the horizontal grounding electrode under the same axial distance, which has good resistance reduction performance. The grounding resistance of the helix grounding electrode with an axial length of $15 \mathrm{~m}$ can reach the horizontal grounding resistance of $34 \mathrm{~m}$, which greatly saves the axial cloth space under the premise of ensuring the same grounding resistance value.
(2)Compared with the horizontal grounding electrode of the same axial length, the electric potential distribution of the helix grounding electrode is smoother and more stable, and the maximum ground potential of the helix grounding electrode is lower, and the soil area of the elevated potential is smaller. It has a better surface potential distribution, which greatly reduces the stride voltage and the contact voltage, and reduces the probability of personal safety accidents.

(3) Thanks to the helix structure of the helix grounding electrode, the distribution of the spill current on the helix grounding electrode is significantly better than that of the horizontal grounding electrode. The helix grounding electrode can achieve a smooth distribution of current in the soil, greatly improving the end effect of the horizontal grounding pole.

In summary, compared with horizontal grounding electrode, the helix grounding electrode can effectively reduce the grounding resistance, improve the surface potential and current distribution, and better adapt to the complex soil environment. In areas with complex terrain, the helix grounding electrode can be used instead of the conventional grounding electrode to achieve safety targets and improve grounding performance.

\section{References}

1. C. J. Poletto, C. L.Doren Van. A high voltage, constant current stimulator for electrocutaneous stimulation through small electrodes. (IEEE Trans Biomed Eng. 46, 8, 1999)

2. Grcev Leonid, Dawalibi Farid. An electromagnetic model for transients in grounding systems. (IEEE T POWER DELIVER. 5, 4 1990)

3. B.Thapar, V.Gerez, A.Balakrishnan, D. Ablank, Evaluation of ground resistance of a grounding grid of any shape. (IEEE T POWER DELIVER. 6, 2 1991)

4. Q. Meng, J. He, F P. Dawalibi, et al. A new method to decrease ground resistances of substation grounding systems in high resistivity regions. (IEEE T POWER DELIVER.14,3 1999)

5. F P. Zupa, J F. Laidig. A Practical Ground Potential Rise Prediction Technique for Power Stations.( IEEE T POWER SYST, 100,1,1980) 